\title{
Thermal conductivity and phonon transport in empty and water-filled carbon nanotubes
}

\author{
John A. Thomas, Ryan M. Iutzi, and Alan J. H. McGaughey* \\ Department of Mechanical Engineering, Carnegie Mellon University, Pittsburgh, Pennsylvania 15213, USA \\ (Received 15 October 2009; revised manuscript received 18 November 2009; published 13 January 2010)
}

\begin{abstract}
The thermal conductivities of empty and water-filled single-walled carbon nanotubes (CNTs) with diameters between 0.83 and $1.36 \mathrm{~nm}$ and lengths ranging from 200 to $1400 \mathrm{~nm}$ are predicted using molecular dynamics simulation. Using a direct application of the Fourier law, we explore the transition to fully diffusive phonon transport with increasing CNT length. For empty CNTs, we find that the CNT length required to obtain fully diffusive phonon transport decreases from $1090 \mathrm{~nm}$ for the $0.83-\mathrm{nm}$-diameter CNT to $510 \mathrm{~nm}$ for the 1.36 nm-diameter CNT. The magnitude of the fully diffusive thermal conductivity also decreases monotonically with increasing CNT diameter. We find that the fully diffusive thermal conductivity of water-filled CNTs is 20\%-35\% lower than that of empty CNTs. By examining the empty and water-filled CNT density of states, we attribute the thermal conductivity reductions to an increase in low-frequency acoustic phonon scattering due to interactions with the water molecules.
\end{abstract}

DOI: 10.1103/PhysRevB.81.045413

PACS number(s): 63.20.-e, 63.22.Gh, 65.40.-b

\section{INTRODUCTION}

The high thermal conductivities of carbon nanotubes (CNTs), measured experimentally ${ }^{1-4}$ and predicted from theory, ${ }^{5-8}$ have generated interest in CNT-based thermal management materials. Most investigations of thermal transport in these materials, however, have focused on their behavior in vacuum. As components of a thermal management device, the atoms in CNTs will exchange energy with atoms and molecules in an adjacent liquid or gas. Results from earlier investigations indicate that the low interaction energy between solid-phase carbon atoms and nonbonded fluid molecules ${ }^{9,10}$ gives rise to velocity slip at the solid/liquid boundary ${ }^{11-14}$ and a large interfacial thermal resistance between CNTs and an adjacent fluid. ${ }^{15-17}$ What remains unclear, however, is how interactions with fluid molecules influence thermal transport in CNTs and modify their thermal conductivities. Understanding how interactions with nonbonded atoms modify phonon transport in a CNT is an important next step in predicting how CNT-based materials will behave in heat transfer applications.

Thermal conductivity, $\mathbf{k}$, is a second-order tensor defined by the Fourier law:

$$
\mathbf{q}^{\prime \prime}=-\mathbf{k} \nabla T,
$$

where $\mathbf{q}^{\prime \prime}$ is the heat flux vector and $\nabla T$ is the spatial temperature gradient. Although defined by this empirical expression, the thermal conductivity of a solid is directly related to the mobility of electrons and phonons. Electronic contributions to the CNT thermal conductivity are generally considered to be negligible. ${ }^{18,19}$ Instead, the high thermal conductivities of CNTs have been attributed to the long phonon mean-free paths associated with low-dimensional (onedimensional or two-dimensional) crystals. ${ }^{20}$

For single-walled CNTs with diameters smaller than $1 \mathrm{~nm}$ and lengths shorter than $O(100 \mathrm{~nm})$, most thermal energy is transported ballistically by acoustic phonons that scatter at the ends of the CNT. ${ }^{21}$ Within this ballistic transport regime, the effective thermal conductivity increases with increasing CNT length as the effects of boundary scattering decrease and additional long-wavelength phonon modes become accessible. ${ }^{21}$ Our use of the term "effective thermal conductivity" emphasizes that the ballistic modes contributing to the heat flux do not follow the Fourier law. ${ }^{7}$ As the CNT length is increased beyond $O(100 \mathrm{~nm})$, an increasing fraction of phonons scatter with other phonons before reaching the ends of the CNT. ${ }^{20,22}$ Eventually, with increasing CNT length, the phonon transport becomes fully diffusive and the thermal conductivity becomes length independent as contributions to thermal transport from ballistic modes become negligible. ${ }^{23}$ The specifics of how this fully diffusive thermal conductivity varies with CNT diameter and how interactions with nonbonded molecules influence this transition are not yet clear. ${ }^{24}$

In this work, we use molecular dynamics (MD) simulation to investigate the fully diffusive thermal conductivity of empty and water-filled single-walled CNTs. We begin by describing our simulation methodology and thermal conductivity prediction procedure. Next, we investigate the variation in thermal conductivity for 200 to 1400 -nm-long CNTs and flat graphene sheets, which can be viewed as infinitediameter single-walled CNTs. For empty CNTs, we find that the fully diffusive thermal conductivity and the length required to obtain fully diffusive phonon transport both decrease with increasing CNT diameter. We attribute this behavior to the increase in acoustic phonon scattering events caused by the larger number of optical phonon modes that exist in larger-diameter CNTs. We find that the magnitude of the fully diffusive thermal conductivity of water-filled CNTs is 20\%-35\% lower than that of the empty CNTs and that the transition to fully diffusive transport occurs at shorter lengths. By comparing the carbon atom density of states (DOS) of empty and water-filled CNTs, we demonstrate that the thermal conductivity reduction is related to the overlap between the vibrational frequencies accessible to the water molecules and the low-frequency acoustic phonon modes in the CNTs.

\section{SIMULATION SETUP}

We simulate thermal transport through empty and waterfilled $0.83,1.10$, and 1.36-nm-diameter CNTs [chirality vec- 


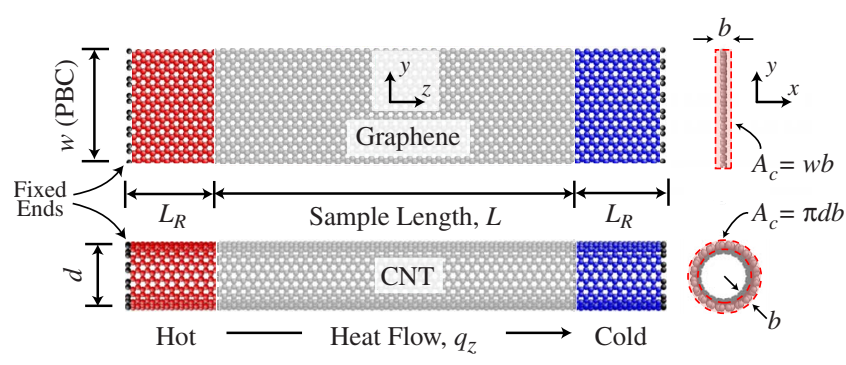

FIG. 1. (Color online) Simulation setup used to predict graphene and CNT thermal conductivities. The dashed lines identify the cross-sectional area, $A_{c}$. The heat flow is introduced by transferring energy from the carbon atoms in the cold reservoir to those in the hot reservoir. The differently colored carbon atoms are used to distinguish the reservoirs from the sample. The reservoir length, $L_{R}$, is $50 \mathrm{~nm}$ and the atoms at the ends of the systems are fixed to prevent sublimation. Periodic boundary conditions are applied in the graphene $y$ direction.

tors of $(6,6),(8,8)$, and $(10,10)]$ and an isolated graphene sheet. As illustrated in Fig. 1, we define the cross-sectional area of the CNTs to be $A_{c}=\pi d b$, where $b(=0.34 \mathrm{~nm})$ is the van der Waals thickness of the CNT surface and $d$ is the CNT diameter. ${ }^{23,25,26}$ We fix one layer of carbon atoms at the ends of the CNT to prevent atoms from sublimating. For graphene, we define the cross-sectional area to be $A_{c}=w b$, where $w$ is the width of the sheet (see Fig. 1). We fix the atoms at the end of the graphene sheet and apply periodic boundary conditions in the $y$ direction. These simulations are thus representative of an infinite graphene sheet and not the graphene nanoribbons studied by others. ${ }^{23,27}$ We note that the systems investigated here are longer than the 10 to $400-n m-$ long systems typically investigated using MD simulation, as summarized by Mingo and Briodo ${ }^{21}$ and Lukes and Zhong. ${ }^{25}$

The number of carbon atoms in the CNT ranges from 29,232 (for the 200-nm-long, 0.83-nm-diameter CNT) to 195,120 (for the 1200-nm-long, 1.10-nm-diameter CNT) and the equilibrium nearest-neighbor distance is $1.42 \AA .{ }^{28}$ Interactions between carbon atoms are modeled using the secondgeneration REBO potential ${ }^{29}$ and interactions between water molecules are modeled using the TIP4P/2005 potential. ${ }^{30}$ The water/carbon interactions are modeled using the Lennard Jones potential of Werder et al. ${ }^{31}$ which was parameterized to reproduce the experimentally observed contact angle of water on graphite. We integrate the equations of motion using a velocity Verlet scheme with a 0.5 fs time step and model the rotational dynamics of the water molecules using the quaternion method. ${ }^{32,33}$

We fill the CNT according to the equilibrium water density, determined by simulating an open-ended sample of each CNT in a liquid water reservoir at a temperature of $298 \mathrm{~K}$ and a pressure of $1 \mathrm{~atm}$. Water molecules were able to freely diffuse across the open ends of the CNT and the density of the water in the reservoir was maintained at $1000 \mathrm{~kg} / \mathrm{m}^{3}$. After a $250 \mathrm{ps}$ initialization period, the average number of molecules enclosed in the CNT became steady, allowing us to determine the equilibrium water density. The water molecules assemble into a single-file molecular chain (0.83-nmdiameter $\mathrm{CNT})$, stacked pentagonal rings $(1.10 \mathrm{~nm})$, or a

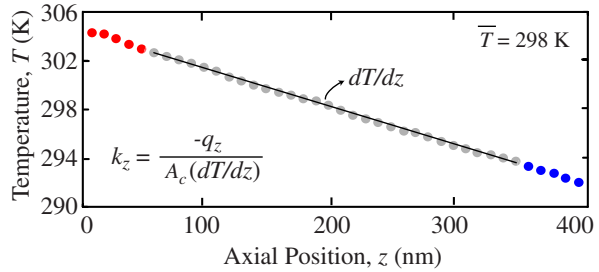

FIG. 2. (Color online) Typical temperature gradient obtained from MD simulation. The thermal conductivity is obtained using Eq. (2). The data here correspond to the 1.36-nm-diameter CNT. The temperature gradient in this trial was $29.5 \pm 1.12 \mathrm{~K} / \mu \mathrm{m}$ (i.e., $3.8 \%$ uncertainty) and the heat flow is $15 \mathrm{nW}$. The uncertainty represents the $95 \%$ confidence interval of the linear regression.

disordered bulk-like liquid $(1.36 \mathrm{~nm}) .{ }^{13}$ As discussed in our previous work, the molecular positional correlations for the chain and ring liquid structures extend 5 to $10 \mathrm{~nm}$ and the structure relaxation times predicted from theory are between $1 \mathrm{~ns}$ and $1 \mathrm{~ms} .{ }^{13}$ These values are much larger than that the 1 $\mathrm{nm}$ position correlation length and $0.5 \mathrm{ps}$ structure relaxation time of bulk water. ${ }^{34}$

We predict the effective axial thermal conductivity, $k_{z}$, of the CNTs and graphene using a one-dimensional form of the Fourier law,

$$
q_{z}=-k_{z} A_{c} \frac{d T}{d z}
$$

where $q_{z}$ is the heat flow in the $z$ direction and $d T / d z$ is the temperature gradient. As shown in Fig. 1, we introduce a heat flow by transferring a known and constant quantity of kinetic energy from carbon atoms in the cold reservoir to those in the hot reservoir at each simulation time step. ${ }^{35,36}$ The total energy of the simulation cell remains constant and corresponds to an average temperature of $298 \mathrm{~K}$. In response to the energy redistribution, however, thermal energy moves from the hot reservoir to the cold reservoir and a temperature gradient is established in the system. We use reservoir lengths of $50 \mathrm{~nm}$ and find that increasing this length has no detectable effect on the predicted thermal conductivity. For graphene, we find that a sample width of $5 \mathrm{~nm}$ is sufficient to eliminate width-related size effects when predicting $k_{z}$.

Each simulation consists of a 10 ns equilibration period (after which the systems reach a steady state), followed by a $10 \mathrm{~ns}$ data collection and averaging period. As depicted in Fig. 2, we specify $d T / d z$ by calculating the temperature of the carbon atoms within 40 subvolumes (i.e., bins) along the sample during the data collection period. Our imposed heat flow of $15 \mathrm{nW}$ introduces a temperature gradient between 25 and $80 \mathrm{~K} / \mu \mathrm{m}$ (depending on the system length and crosssectional area). The temperature within each bin is related to the average kinetic energy of the carbon atoms. Thus, with increasing CNT diameter and length, a larger number of carbon atoms are in each bin and the uncertainty in the temperature gradient decreases from $10 \%$ in the empty 200-nm-long, 0.83 -nm-diameter CNT to less than $2 \%$ in the empty 1200 nm-long, 1.10-nm-diameter CNT. The uncertainty in the temperature gradient for all the water-filled CNTs is less than $2 \%$. Consistent with systems in a linear-response regime, we 


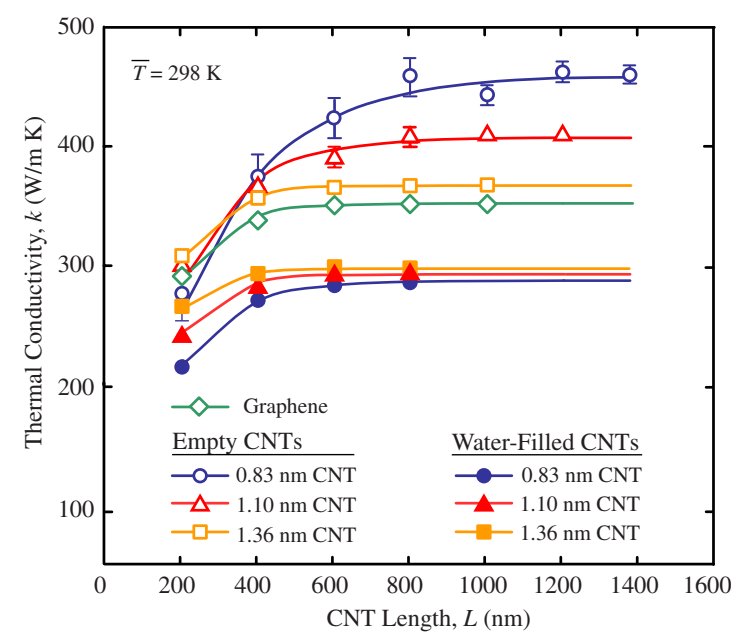

FIG. 3. (Color online) Variation in thermal conductivity with length for empty CNTs, water-filled CNTs, and graphene. The diameter of each CNT is given in the legend and each data set is fit to Eq. (3). The error bars for $k_{z}$ are associated with uncertainty in specifying $d T / d z$. For points without error bars, the uncertainty in the thermal conductivity is comparable to the size of the data point.

find that the predicted thermal conductivity is not affected by small changes in the magnitude of the heat flow.

\section{EMPTY CNT THERMAL CONDUCTIVITY}

In Fig. 3, we present the variation in thermal conductivity with length for the empty $0.83,1.10$, and $1.36 \mathrm{~nm}$-diameter CNTs and for graphene. From this data, we observe the transition from a regime where the thermal conductivity is length dependent to a regime where it is length-independent and the phonon transport is fully diffusive. To quantify the transition from ballistic to diffusive phonon transport, we fit the thermal conductivity data to the empirical function

$$
k_{z}=k_{z, \infty}\left[1-\exp \left(-\frac{L}{L_{c}}\right)\right],
$$

where $k_{z, \infty}$ is the fit fully diffusive thermal conductivity, $L$ is the sample length, and $L_{c}$ is a fit length that describes the transition. We define the quantity $L_{99}$ to be the length at which $k_{z}=0.99 k_{z, \infty}$ such that

$$
L_{99} \equiv \ln (100) L_{c}=4.605 L_{c} .
$$

Another procedure for extracting $k_{z, \infty}$, as proposed by Schelling et al. ${ }^{37}$ incorporates the effects of boundary scattering into the phonon mean-free path using the Matthiessen rule. ${ }^{38}$ The Matthiessen rule, when combined with the kinetic theory expression for thermal conductivity, ${ }^{39}$ predicts that $1 / k_{z}$ will be linearly related to $1 / L$ such that finite-size effects can be eliminated using a linear extrapolation (e.g., $1 / L \rightarrow 0)$. Although this procedure has been successfully applied to three-dimensional solid systems, ${ }^{36,37,40}$ the relationship between $1 / k_{z}$ and $1 / L$ for the CNTs considered here is nonlinear. A direct extrapolation to an infinite system length is therefore not possible. We hypothesize that the primary reason for this breakdown of the linear extrapolation proce- (a)
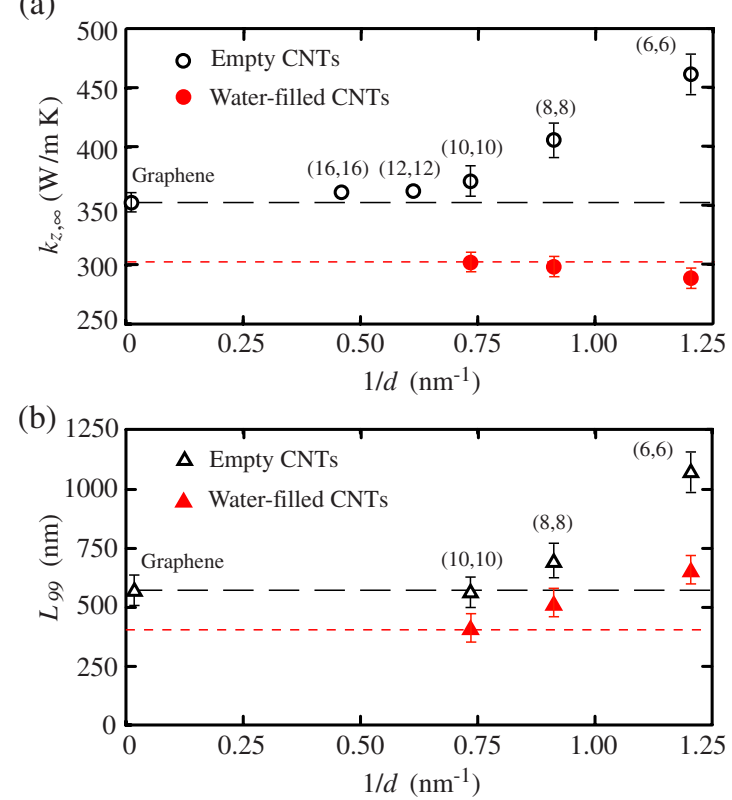

FIG. 4. (Color online) Variation in (a) $k_{z, \infty}$ and (b) $L_{99}$ with the inverse of diameter for empty and water-filled CNTs. The chirality vectors for each CNT are used to label the data points. We also include $k_{z, \infty}$ for empty $1.63-\mathrm{nm}$-diameter $\left(1 / d=0.61 \mathrm{~nm}^{-1}\right)$ and 2.17-nm-diameter $\left(1 / d=0.46 \mathrm{~nm}^{-1}\right) \mathrm{CNTs}$, which are predicted using a 600-nm-long CNT sample (Ref. 42). The horizontal longdashed lines indicate $k_{z, \infty}$ and $L_{99}$ for the flat graphene sheet $(1 / d$ $=0$ ). The horizontal short-dashed lines indicate our estimated values for $k_{z, \infty}$ and $L_{99}$ for water/graphene systems based on analysis of the CNT density of states (see Sec. IV). The error bars indicate the standard error calculated from a regression analysis of Eq. (3).

dure is the assumption of a single phonon mean-free path in the kinetic theory expression for thermal conductivity. ${ }^{41}$ This approximation, which assumes that every phonon travels the same distance on average before scattering, is poor for CNTs and graphene, where the low-frequency acoustic phonon modes have mean-free paths several orders-of-magnitude larger than those of the other acoustic modes. ${ }^{5}$

In Figs. 4(a) and 4(b) we present the variation in $k_{z, \infty}$ and $L_{99}$ with the inverse of the CNT diameter. Included in the plots are additional $k_{z, \infty}$ values for empty $1.63 \mathrm{~nm}$ and 2.17 nm-diameter CNTs that we predict from simulations of 600nm-long samples. ${ }^{42}$ For the empty CNTs, we find that both $k_{z, \infty}$ and $L_{99}$ decrease with increasing CNT diameter and approach the value we predict for graphene. To understand this behavior, note that the high thermal conductivity of CNTs is related to the long-wavelength acoustic phonon modes accessible to the system. ${ }^{20}$ With increasing CNT diameter, the number of acoustic phonon modes does not change but optical phonon modes are created due to the increased number of atoms in the unit cells. Since the optical phonons in CNTs typically have small mean-free paths, their mode-by-mode contributions to thermal conductivity are small. ${ }^{43}$ As suggested by the Fermi golden rule for phonon scattering, ${ }^{44}$ however, the additional optical modes in larger-diameter CNTs present additional multi-phonon scattering channels that shorten the acoustic phonon mean-free paths. ${ }^{45,46}$ These additional interactions lower the fully diffusive thermal con- 
ductivity in the larger-diameter CNTs and, due to the shorter acoustic phonon mean-free paths, shorten the CNT length required to obtain fully diffusive transport. ${ }^{47}$

The data presented in Figs. 4(a) and 4(b) are consistent with the MD simulation results of Shiomi and Maruyama, who, also using the REBO potential, found that $k_{z}$ for a 1000-nm-long, $0.41 \mathrm{~nm}$-diameter CNTs is higher than that for a 0.68 -nm-diameter CNTs with the same length. ${ }^{23}$ Our predicted fully diffusive thermal conductivity of $355 \mathrm{~W} / \mathrm{m}-\mathrm{K}$ for the 1.36-nm-diameter CNT is consistent with the MD simulation predictions of Padgett and Brenner, ${ }^{26}$ who modeled the same CNT using the REBO potential and predicted a fully diffusive thermal conductivity of $350 \mathrm{~W} / \mathrm{m}-\mathrm{K}$. We note that the thermal conductivities of graphitic materials predicted using the REBO potential are lower than those measured from experiments, which range from 2,000 to $10,000 \mathrm{~W} / \mathrm{m}-\mathrm{K} .{ }^{3,4,25,48}$ This behavior is in contrast to the Tersoff potential, ${ }^{49}$ which tends to overestimate the thermal conductivity of graphitic materials. ${ }^{5}$ Our predictions from anharmonic lattice dynamics calculations indicate that a fully quantum mechanical treatment of the phonon transport in our CNTs at a temperature of $298 \mathrm{~K}$ changes the magnitude of the fully diffusive thermal conductivity by less than $5 \% .{ }^{50}$

\section{WATER-FILLED CNT THERMAL CONDUCTIVITY}

We predict the thermal conductivity of water-filled CNTs using the same heat flux method used to investigate empty CNTs. The water-filled CNTs are filled with the number of water molecules corresponding to the equilibrium density and span the entire simulation cell. No water is present outside the CNT. After the $10 \mathrm{~ns}$ equilibration period, we find that the axial temperature profiles in the CNT and in the water are identical. This expected steady-state condition implies that the net energy transfer between the two materials is zero but that thermal energy will be transferred from the hot reservoir to the cold reservoir through both materials. Our preliminary investigations indicate that the thermal conductivity of the ordered water in these CNT is approximately $1 \mathrm{~W} / \mathrm{m} \mathrm{K} .{ }^{51,52}$ Since the water and CNTs in our study have comparable cross-sectional areas, the relationship between their thermal conductivities implies that a negligible fraction of the total heat transfer through the composite occurs through the water (i.e., the water has a negligible thermal conductance). As discussed below, however, interactions between the carbon atoms and the water molecules influence phonon transport in the CNT and cannot be ignored.

In Fig. 3, we compare the thermal conductivities of the water-filled CNTs to those of the empty CNTs. Although simulating longer water-filled CNTs is computational impractical, the data presented here are sufficient to illustrate the transition to fully diffusive transport. We calculate $k_{z, \infty}$ and $L_{99}$ using Eqs. (3) and (4) and report these values in Figs. 4(a) and 4(b). Three trends are apparent: First, the fully diffusive thermal conductivities of the water-filled CNTs are $20 \%$ to $35 \%$ lower than those of the empty CNTs. Second, the CNT length required to obtain fully diffusive thermal transport in water-filled CNTs is shorter than that required for empty CNTs. Third, unlike in empty CNTs, where the (a)

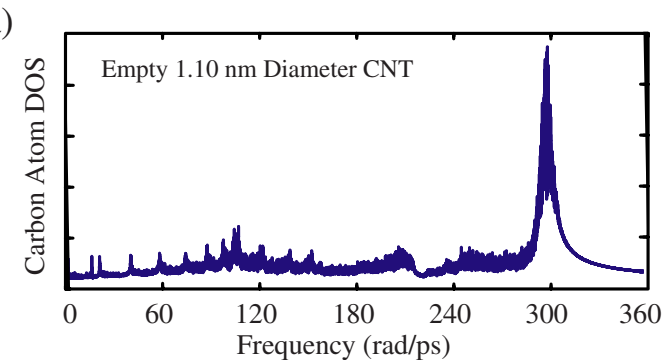

(b)

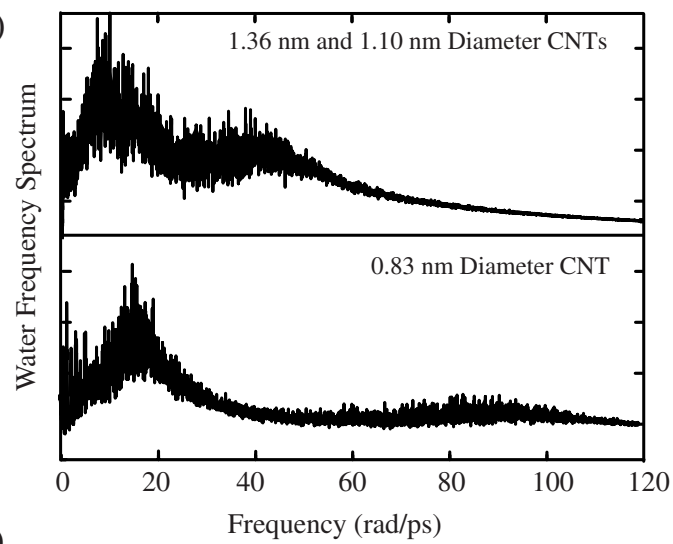

(c)

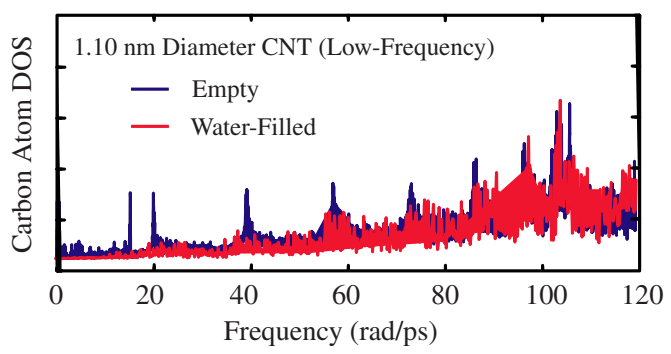

FIG. 5. (Color online) (a) DOS for the 1.10-nm-diameter empty CNT. The DOS for 0.83 and 1.36-nm-diameter CNTs have similar features. (b) Vibrational spectra of water molecules inside 1.36-, 1.10-, and 0.83-nm-diameter CNTs. The spectra inside the 1.36- and 1.10-nm-diameter CNTs are similar to what we predict for bulk water. (c) Low-frequency DOS for the carbon atoms in the empty and water-filled 1.10-nm-diameter CNT. As the magnitude of the water molecule spectrum decreases with increasing frequency, the DOS for carbon atoms in the empty and water-filled CNTs converge.

fully diffusive thermal conductivity decreases with increasing diameter, the thermal conductivity of the water-filled 0.83-nm-diameter CNT is lower than that of the water-filled 1.36 and 1.10-nm-diameter CNTs (which are the same to within the prediction uncertainty).

To understand the mechanisms responsible for these trends, we plot in Fig. 5(a) the DOS for carbon atoms in the empty 1.10-nm-diameter CNT predicted from the Fourier transform of the carbon atom velocity autocorrelation function. ${ }^{53}$ The DOS was obtained from equilibrium simulation (no heat flux) of a 200-nm-long CNT fragment with fixed ends at a temperature of $298 \mathrm{~K}$ in the microcanonical ensemble. The same DOS is predicted from simulations using a periodic boundary condition in the axial direction. For both the fixed-end and periodic systems, we find that increasing the CNT length does not change the position or relative 
magnitudes of the peaks in the DOS. The DOS for the empty 0.83 and 1.36-nm-diameter CNTs are similar to that for the 1.10-nm-diameter CNT, although the larger number of optical modes in the larger-diameter CNTs cause the number of frequency peaks to increase with increasing diameter. As discussed by others, ${ }^{5,20,21}$ a large part of the CNT thermal conductivity comes from the acoustic phonon modes. An inspection of CNT dispersion curves ${ }^{18,54}$ indicates that these acoustic modes have frequencies less than $120 \mathrm{rad} / \mathrm{ps}$.

In Fig. 5(b), we plot the vibrational frequencies accessed by the water molecules inside each CNT. This spectrum is calculated from the Fourier transform of the water molecule velocity autocorrelation function. The vibrational spectra for water inside the 1.36 and 1.10-nm-diameter CNTs are both similar to that of bulk water and indicate that the molecules inside the CNT vibrate primarily with frequencies below 60 $\mathrm{rad} / \mathrm{ps}$. Inside the 0.83-nm-diameter CNT, where water molecules assemble into a one-dimensional hydrogen-bonded water wire, the range of accessible frequencies extends up to $100 \mathrm{rad} / \mathrm{ps}$. We believe that the extended range of frequencies accessible to these water molecules is related to the stability and correspondingly long structure relaxation time $(\sim 1 \mathrm{~ms})$ of the water wire. ${ }^{55}$

In Fig. 5(c), we compare the low-frequency DOS for carbon atoms in the empty 1.10 -nm-diameter CNT to what we predict for the water-filled 1.10-nm-diameter CNT. Changes in the DOS are particularly evident in the sub-40 rad/ps frequency range, where peaks present in the empty CNT DOS are missing from the water-filled CNT DOS. As shown in Fig. 5(b), this frequency range is highly populated by the water molecules inside the CNT, suggesting that collisions between water molecules and the carbon surface scatter the low-frequency acoustic phonons traveling through the CNT. As the magnitude of the water molecule spectrum decreases with increasing frequency, the DOS for carbon atoms in the empty and water-filled CNTs converge. This finding is consistent with theoretical investigations into heat transfer across a CNT/liquid octane interface, which indicate that liquid polymers exchange energy primarily with CNT phonon modes with comparable vibrational frequencies. ${ }^{15,16}$

By definition, the area under the CNT DOS is equal to the total number of phonon modes accessible to the carbon atoms. This number is the same for both the empty and waterfilled CNTs, meaning that the missing peaks in the waterfilled CNT DOS indicate a broadening (but not elimination) of the phonon modes accessible to the empty CNTs. Molecular dynamics-based investigations of thermal boundary resistance between CNTs and liquid or solid argon indicate that this broadening is primarily caused by phonon linewidth collision broadening. ${ }^{56}$ This behavior is explained as follows: the phonon linewidth, which is inversely proportional to the phonon relaxation time, describes how the actual atomic vibrational frequencies deviate from the non-interacting normal modes of vibration. In empty CNTs, the phonon linewidth is related to the number of phonon-phonon scattering interactions that arise from anharmonicities in the potential energy landscape. ${ }^{57}$ In water-filled CNTs, interactions between the phonons and nonbonded molecules further perturb the carbon atom potential energy landscape. This behavior increases the range of atomic vibrational frequencies ac- cessed by the carbon atoms in specific modes, further increasing the number of phonon-phonon interactions within the crystal. Collision broadening due to interactions with the water molecules therefore smears phonon modes over a wider range of vibration frequencies, reducing the height of individual peaks in the carbon atom DOS, and reducing the corresponding low-frequency phonon lifetimes.

Based on the above discussion, we believe that lowfrequency acoustic phonon scattering due to interactions with water molecules is primarily responsible for the reduced $k_{z, \infty}$ in water-filled CNTs [see Fig. 4(a)]. In support of this argument, we note that the increased range of vibrational frequencies accessible to the water molecules in the $0.83-\mathrm{nm}$ diameter CNT cause them to influence more phonon modes than do the water molecules in larger-diameter CNTs. Since the thermal energy transported by these higher-frequency modes is smaller than that transported by the low-frequency acoustic phonons, however, the additional reduction in thermal conductivity is small. This behavior also suggests that, given the weak dependence of $k_{z, \infty}$ on diameter for the waterfilled CNTs, the thermal conductivity of graphene in bulklike water will be similar to $k_{z, \infty}$ for the water-filled 1.36-nmdiameter CNT [as indicated in Fig. 4(b)]. Additionally, since interactions between CNTs and water molecules outside the CNT are unaffected by changes in the CNT surface curvature, ${ }^{58}$ we believe that $k_{z, \infty}$ for CNTs surrounded by liquid water (i.e., water only outside the CNT) will be similar to that predicted here for the water-filled 1.36 and $1.10-\mathrm{nm}$ diameter CNTs.

\section{SUMMARY}

We used MD simulation to examine the transition to fully diffusive phonon transport in empty and water-filled CNTs and in graphene. For empty CNTs, as shown in Fig. 3, we find that the magnitude of the fully diffusive thermal conductivity and the length required to obtain fully diffusive transport both decrease with increasing CNT diameter. We attribute this behavior to the higher number of acoustic phonon mode scattering events due to the larger number of optical phonon modes accessible to larger-diameter CNTs. As presented in Fig. 4, we find that the fully diffusive thermal conductivity of water-filled CNTs is $20 \%-35 \%$ lower than that of empty CNTs and only weakly dependent on CNT diameter. By examining the carbon atom DOS and water molecule vibrational spectra presented in Fig. 5, we showed that water molecules only scatter phonons with comparable vibrational frequencies. This finding indicates that lowfrequency phonon scattering due to interactions with water molecules is primarily responsible for the reduction in the CNT thermal conductivity. Better intermolecular potential functions are needed, however, to make a more quantitative comparison between simulation predictions and future experimental measurements.

Although focused on water/CNT systems, the results presented here can be extended to other solid/liquid composite systems. Most importantly, reductions in the thermal conductivity of a solid in a fluid will be related to the overlap between its phonon frequencies and the vibrational frequen- 
cies of the adjacent nonbonded molecules. This relationship is an important feature of composite systems and indicates that not all phonons are influenced equally by interactions with nonbonded molecules. ${ }^{5}$ In a real system, compared to the classical MD system modeled here, consideration must also be given to the phonon quantum occupation number. For water-filled CNTs, the phonon modes that overlap with the water molecule vibrational frequencies are highly populated at room temperature, so that we believe our results are representative of an actual water/CNT composite. In other composite systems, the relationship between temperature, phonon mode population, and liquid vibrational spectrum may need to be considered explicitly. Finally, it is important to note that the influence of solid/liquid interactions on the solid thermal conductivity is also related to the ratio of the solid surface area to solid volume. When this ratio is large (as in a single-walled CNT), the influence of nonbonded molecules on phonon transport is appreciable. Conversely, when this ratio is small, surface effects will be negligible and phonon transport through the solid will be minimally affected by interactions with nonbonded atoms.

\section{ACKNOWLEDGMENTS}

We thank Eric Landry and Joseph Turney at Carnegie Mellon University for helpful discussions. *mcgaughey@cmu.edu

${ }^{1}$ J. Hone, M. C. Llaguno, N. M. Nemes, and A. T. Johnson, Appl. Phys. Lett. 77, 666 (2000).

${ }^{2}$ J. Hone, M. Whitney, C. Piskoti, and A. Zettl, Phys. Rev. B 59, R2514 (1999).

${ }^{3}$ E. Pop, D. Mann, Q. Wang, K. Goodson, and H. Dai, Nano Lett. 6, 96 (2006).

${ }^{4}$ C. Yu, L. Shi, Z. Yao, D. Li, and A. Majumdar, Nano Lett. 5, 1842 (2005).

${ }^{5}$ D. Donadio and G. Galli, Phys. Rev. Lett. 99, 255502 (2007).

${ }^{6}$ J. F. Moreland, J. B. Freund, and G. Chen, Nanoscale Microscale Thermophys. Eng. 8, 61 (2004).

${ }^{7}$ J. Shiomi and S. Maruyama, Phys. Rev. B 73, 205420 (2006).

${ }^{8}$ S. Maruyama, Nanoscale Microscale Thermophys. Eng. 7, 41 (2003).

${ }^{9}$ X. Zhao and J. K. Johnson, Mol. Simul. 31, 1 (2005).

${ }^{10} \mathrm{G}$. Jenness and K. D. Jordan, J. Phys. Chem. C 113, 10242 (2009).

${ }^{11}$ J. K. Holt, H. G. Park, Y. Wang, M. Stadermann, A. B. Artyukhin, C. P. Grigoropoulos, A. Noy, and O. Bakajin, Science 312, 1034 (2006)

${ }^{12}$ J. A. Thomas and A. J. H. McGaughey, Nano Lett. 8, 2788 (2008).

${ }^{13}$ J. A. Thomas and A. J. H. McGaughey, Phys. Rev. Lett. 102, 184502 (2009).

${ }^{14}$ M. Whitby, L. Cagnon, M. Thanou, and N. Quirke, Nano Lett. 8, 2632 (2008).

${ }^{15}$ S. Shenogin, A. Bodapati, L. Xue, R. Ozisik, and P. Keblinski, Appl. Phys. Lett. 85, 2229 (2004).

${ }^{16}$ S. Shenogin, L. Xue, R. Ozisik, P. Keblinski, and D. G. CahillJ. Appl. Phys. 95, 8136 (2004).

${ }^{17}$ S. T. Huxtable, D. G. Cahill, S. Shenogin, L. Xue, R. Ozisik, P. Barone, M. Usrey, M. S. Strano, G. Siddons, M. Shim, and P. Keblinski, Nature Mater. 2, 731 (2003).

${ }^{18}$ J. Hone, in Dekker Encyclopedia of Nanoscience and Nanotechnology, edited by J. A. Schwarz, C. I. Contescu, and K. Putyera (Dekker, New York, 2004), pp. 603-610.

${ }^{19}$ L. X. Benedict, S. G. Louie, and M. L. Cohen, Solid State Commun. 100, 177 (1996).

${ }^{20}$ N. Mingo and D. A. Broido, Nano Lett. 5, 1221 (2005).

${ }^{21}$ N. Mingo and D. A. Broido, Phys. Rev. Lett. 95, 096105 (2005).

${ }^{22}$ P. Heino, Microsyst. Technol. 15, 75 (2009).
${ }^{23}$ J. Shiomi and S. Maruyama, Jpn. J. Appl. Phys. 47, 2005 (2008).

${ }^{24}$ J. Shiomi and S. Maruyama, Nanotechnology 20, 055708 (2009).

${ }^{25}$ J. R. Lukes and H. Zhong, ASME J. Heat Transfer 129, 705 (2007).

${ }^{26}$ C. W. Padgett and D. W. Brenner, Nano Lett. 4, 1051 (2004).

${ }^{27}$ J. Hu, X. Ruan, and Y. P. Chen, Nano Lett. 9, 2730 (2009).

${ }^{28}$ P. J. F. Harris, Carbon Nanotube Science (Cambridge University Press, New York, 2009).

${ }^{29}$ D. W. Brenner, O. A. Shenderova, J. A. Harrison, S. J. Stuart, B. Ni, and S. B. Sinnott, J. Phys.: Condens. Matter 14, 783 (2002).

${ }^{30}$ J. L. F. Abascal and C. Vega, J. Chem. Phys. 123, 234505 (2005).

${ }^{31}$ T. Werder, J. H. Walther, R. L. Jaffe, T. Halicioglu, and P. Koumoutsakos, J. Phys. Chem. B 107, 1345 (2003).

${ }^{32}$ M. P. Allen and D. J. Tildesley, Computer Simulation of Liquids (Clarendon Press, Oxford, 1987).

${ }^{33}$ C. F. F. Karney, J. Mol. Graph. Model. 25, 595 (2007).

${ }^{34}$ A. K. Soper, J. Chem. Phys. 101, 6888 (1994).

${ }^{35}$ T. Ikeshoji and B. Hafskjold, Mol. Phys. 81, 251 (1994).

${ }^{36}$ E. S. Landry, M. I. Hussein, and A. J. H. McGaughey, Phys. Rev. B 77, 184302 (2008).

${ }^{37}$ P. K. Schelling, S. R. Phillpot, and P. Keblinski, Phys. Rev. B 65, 144306 (2002).

${ }^{38}$ J. M. Ziman, Electrons and Phonons: The Theory of Transport Phenomena in Solids (Clarendon Press, Oxford, 1960).

${ }^{39}$ N. W. Ashcroft and N. D. Mermin, Solid State Physics (Saunders, Fort Worth, 1976).

${ }^{40}$ Y. Chen, D. Li, J. R. Lukes, Z. Ni, and M. Chen, Phys. Rev. B 72, 174302 (2005).

${ }^{41}$ D. P. Sellan, E. S. Landry, J. E. Turney, A. J. H. McGaughey, and C. H. Amon (unpublished).

${ }^{42}$ Based on the $L_{99}$ values for the 1.36-nm-diameter CNT and for graphene, we believe that the thermal conductivities predicted from simulations of 600-nm-long, 1.63- and 2.17-nm-diameter CNTs represent the fully diffusive thermal conductivity.

${ }^{43}$ J. Yang, in Thermal Conductivity: Theory, Properties and Applications, edited by T. M. Tritt (Kluwer Academic, New York, 2004), pp. 1-20.

${ }^{44}$ A. A. Maradudin and A. E. Fein, Phys. Rev. 128, 2589 (1962).

${ }^{45}$ G. P. Srivastava, in High Thermal Conductivity Materials, edited 
by S. L. Shinde and J. S. Goela (Springer, Boca Raton, 2006), pp. 1-35.

${ }^{46}$ G. P. Srivastava, J. Phys. Chem. Solids 41, 357 (1980).

${ }^{47}$ L. Lindsay, D. A. Broido, and N. Mingo, Phys. Rev. B 80, 125407 (2009).

${ }^{48}$ A. A. Balandin, S. Ghosh, W. Bao, I. Calizo, D. Teweldebrhan, F. Miao, and C. N. Lau, Nano Lett. 8, 902 (2009).

${ }^{49}$ J. Tersoff, Phys. Rev. B 39, 5566 (1989).

${ }^{50}$ J. E. Turney, Ph. D Thesis, Carneige Mellon University, Pittsburgh, PA.

${ }^{51}$ J. A. Thomas, R. M. Iutzi, and A. J. H. McGaughey, Proceedings of the SHTC (ASME, New York, 2009), Paper No. HT200988029.

${ }^{52}$ W. Evans, J. Fish, and P. Keblinski, J. Chem. Phys. 126, 154504
(2007).

${ }^{53}$ M. T. Dove, Introduction to Lattice Dynamics (Cambridge University Press, Cambridge, 1993).

${ }^{54}$ R. Saito, G. Dresselhaus, and M. S. Dresselhaus, Physical Properties of Carbon Nanotubes (Imperial College Press, London, 2003).

${ }^{55}$ J. Kofinger, G. Hummer, and C. Dellago, Proc. Natl. Acad. Sci. U.S.A. 105, 13218 (2008).

${ }^{56}$ C. F. Carlborg, J. Shiomi, and S. Maruyama, Phys. Rev. B 78, 205406 (2008).

${ }^{57}$ A. Debernardi, S. Baroni, and E. Molinari, Phys. Rev. Lett. 75, 1819 (1995).

${ }^{58}$ J. A. Thomas and A. J. H. McGaughey, J. Chem. Phys. 128, 084715 (2008). 